\title{
Reflexiones del programa de extensión docente English for Kids de la Sede del Pacífico de la Universidad de Costa Rica
}

\section{A reflection on the teaching program English for Kids, from the Pacific Regional Center, University of Costa Rica}

\author{
Rosberly López Montero ${ }^{1}$ \\ Universidad de Costa Rica \\ Sede del Pacífico \\ Puntarenas, Costa Rica \\ rosberly@yahoo.es \\ ORCID: http://orcid.org/0000-0002-2000-4856 \\ Karla Rodríguez-Chaves ${ }^{2}$ \\ Universidad de Costa Rica \\ Sede del Pacífico \\ Puntarenas, Costa Rica \\ karlapam05@hotmail.com \\ ORCID: http://orcid.org/0000-0003-2392-8795
}

Recibido: 21 mayo 2015 Aceptado: 16 junio 2016 Corregido: 15 agosto 2016

\begin{abstract}
Resumen: El presente ensayo tiene como objetivo dar a conocer los aportes del programa English for Kids, del proyecto de extensión docente "Idiomas para la Comunicación Internacional" de la Sede Regional del Pacífico, Arnoldo Ferreto Segura de la Universidad de Costa Rica. Se toma en cuenta el efecto de las giras académicas como estrategia didáctica y los resultados de la evaluación de actividades de extensión docente realizada tanto a padres y madres de familia como al estudiantado. Se destaca el papel de las giras como una estrategia efectiva para practicar el idioma fuera del aula, así como los efectos percibidos desde la perspectiva de las personas participantes a partir de los cursos de inglés que han recibido en el programa. Los resultados demuestran que el proyecto ha contribuido al deseo existente en la población de adquirir un nuevo idioma desde una edad temprana.
\end{abstract}

Palabras clave: Bilingüismo, educación, inglés, niñez, gira académica.

\begin{abstract}
This paper intends to highlight the contribution of the project Languages for International Communication of the Pacific Regional Center of the University of Costa Rica and its program English for Kids. Thus, the program analyzes two main components: the effect of the academic field trip as a didactic strategy and the results of the evaluation for extension activities carried out to both parents and students. The paper highlights the role of field trips as an effective strategy to practice the language outside the classroom, as well as the effects obtained by the participants thanks to the English courses the program has offered. The results show that the project has contributed to the existing desire of the population to acquire another language at an early age.
\end{abstract}

Keywords: Bilingualism, education, English, childhood, academic field trip.

1 Licenciada en la Enseñanza del Inglés. Máster en Lingüística de la Universidad de Costa Rica. Docente del área de inglés de la Sede del Pacífico de la Universidad de Costa Rica. Coordinadora del proyecto de extensión docente "Idiomas para la Comunicación internacional" de la Sede del Pacífico de la Universidad de Costa Rica.

2 Bachiller en Enseñanza del Inglés de la Universidad de Costa Rica. Profesora de inglés en el proyecto Idiomas para la Comunicación Internacional de la Sede del Pacífico de la Universidad de Costa Rica. Encargada del programa English for Kids de dicho proyecto. 


\section{Introducción}

Actualmente el bilingüismo es una herramienta que brinda posibilidades para sobresalir en la sociedad, tener mejores oportunidades laborales y ostentar mayores posibilidades de explorar el mundo, además de otra serie de beneficios. Por ejemplo, Vásquez-Carranza (2009) apunta que el desarrollo cognitivo de las personas bilingües sufre un impacto positivo significativo; aunado a esta idea, Hasbún-Hasbún (2007) destaca la importancia de contar con programas nacionales para comunicarse en diversas lenguas para que, de esta manera, la ciudadanía pueda formar parte de la comunidad global. A pesar de que existen también posturas que argumentan que el bilingüismo puede ser perjudicial en la edad escolar, ya que las personas hablantes tienden a confundir el uso de ambas lenguas en un mismo contexto (Montrul, 2013), tales posturas pierden fuerza al considerar que en dichos casos el bilingüismo no es analizado como un fenómeno en sí mismo, sino que se tiende a comparar con una población monolingüe, lo que proporciona una visión fraccionada del bilingüismo (Montrul, 2013).

No obstante, ese panorama, el aprendizaje de otras lenguas se convierte en una tendencia en crecimiento, marcando una evidente importancia de fomentar la educación bilingüe desde edades tempranas. En este sentido, el que los niños y las niñas expandan sus conocimientos aprendiendo una nueva lengua, además de su idioma materno, les facilita el acceso a estos beneficios.

Específicamente la enseñanza del inglés en Costa Rica forma parte de las políticas educativas, debido al contexto económico en que se encuentra el país: muchas empresas multinacionales se instalan en territorio costarricense y requieren de personal capacitado en este idioma extranjero (Marín-Arroyo, 2012). Además, según Harmer (2007), el idioma inglés es hablado, por lo menos, por un cuarto de la población mundial y es, además, reconocido como la lengua franca, que -según este mismo autor- es la lengua en común entre dos personas que no hablan el mismo idioma. Es decir, esto es importante por indicar que la lengua inglesa es utilizada en distintos contextos comunicativos alrededor del mundo.

En este ensayo se parte de la importancia del bilingüismo desde la niñez temprana como herramienta eficaz en la consecución de metas profesionales y sociales por parte del individuo. Dentro de esta postura, Zhizhko y Koudrjavtseva-Hentschel (2011) comentan que "la mayoría de los niños bilingües perciben el lenguaje -materno y extranjero- como instrumento de integración a determinada sociedad de manera más intensa y viva que sus compañeros monolingües" (p. 54).

Retomando palabras de Harmer (2007), la mayoría de personas usuarias competentes del idioma inglés lo han aprendido como lengua extranjera, lo cual señala la importancia de aprender este idioma además de la lengua nativa. En vista de esta realidad, cada vez más centros educativos ofrecen una opción de enseñanza del inglés enfocada en los sujetos aprendices más jóvenes; sin embargo, en el caso de Costa Rica, muchas de estas propuestas se concentran en el Valle Central del país, por lo que es importante que existan iniciativas de enseñanza del idioma inglés para la niñez de las áreas periféricas. Por esta razón, el presente documento pretende ahondar en el trabajo realizado desde el proyecto "Idiomas para la Comunicación Internacional" y su programa English for Kids de la Sede del Pacífico de la Universidad de Costa Rica. El programa le posibilita a la niñez puntarenense desarrollar el conocimiento de esta lengua y beneficiarse del bilingüismo desde edades tempranas; 
igualmente incrementa la motivación del estudiantado durante su proceso de aprendizaje y propicia el desarrollo de una actitud positiva hacia la lengua, favoreciendo que las experiencias con esta lengua a lo largo de su vida futura, sean también positivas.

\section{Antecedentes}

La provincia de Puntarenas, en Costa Rica, se sitúa en el Pacífico Central del país. De acuerdo con el Instituto Nacional de Estadística y Censos, la cabecera de esta provincia tiene una población de 115019 personas (INEC, Censo 2011), lo cual lo convierte en el cantón más poblado de Puntarenas. Los proyectos de extensión universitaria contribuyen a que regiones como estas, fuera de las zonas de concentración de mejores oportunidades de estudio y empleo, fortalezcan el crecimiento académico de sus habitantes y "contribuir al desarrollo de capacidades en las comunidades con las que se relaciona" (Universidad de Costa Rica, s. f., Acción Social, 1 Párr.). La capacidad de comunicarse en una lengua extranjera es lo que impulsa el programa English for Kids que imparte cursos de inglés a niños y niñas a partir de los tres años de edad, con lo cual se convierte en un programa innovador en la región del Pacífico Central costarricense, región que solía carecer de un programa de educación abierta continua en la enseñanza del inglés para la población infantil.

El programa English for Kids, perteneciente al proyecto de Extensión Docente ED-2884, "Idiomas para la Comunicación Internacional", pretende ser un impulsor de oportunidades a partir del conocimiento de una nueva lengua. Desde su inicio en el año 2013, su acogida en la comunidad ha sido perceptible por medio de la respuesta de la población y los bajos índices de deserción estudiantil, ya que la mayor parte del estudiantado continúa con el programa completo, que en total tiene año y medio de duración.

El contar con la opción de un programa de enseñanza del inglés para aquellos y aquellas estudiantes que ya se encuentran dentro de la educación formal, además de la instrucción recibida en las instituciones educativas, es una manera de alivianar las exigencias laborales que se encontrarán en el futuro. Por tanto, "un programa de inglés, en la modalidad de educación abierta, contribuye, de manera sustantiva, a solventar las faltantes a nivel regional y nacional, en cuanto al recurso humano bilingüe" (López-Montero, 2014, p. 137), por lo que el aprendizaje de una lengua extranjera a partir de edades tempranas, provocará también un impacto social, además del individual.

Este programa está conformado por seis niveles; cada nivel con una duración de veinte horas; divididas en sesiones semanales de dos horas. La razón principal de este número de horas por semana es porque es considerado el tiempo suficiente para que la población más pequeñina participe de la clase activamente sin llegar a aburrirse, un período de mayor cantidad de horas podría significar no solamente aburrimiento, sino también cansancio, y dificultar el aprendizaje. No obstante, si consideramos la poca exposición a la lengua meta por parte de esta población cuando se encuentran fuera del aula, el tiempo efectivo de clase es poco, por lo que el objetivo del programa de preescolar se enfoca en asentar las bases para el aprendizaje de la lengua y que, de este modo, sea más fácil aprenderla en el futuro por lo que al final del programa se pretende que el estudiantado más joven domine vocabulario básico, 
reconozca estructuras y se desempeñe en interacciones cortas con sus pares, lo anterior a través de actividades de mucha repetición y de aprendizaje lúdico, junto con pequeños proyectos extra clase.

Para los chicos y chicas en edad escolar, de siete a doce años, las dos horas de clase se enfocan en realizar actividades orales con el propósito de que refuercen los contenidos vistos en sus escuelas, pues, aunque inglés es una materia básica en la educación primaria costarricense desde el año de 1997 (Córdoba, Coto y Ramírez, 2005), este proyecto propicia mayor espacio para el desarrollo de las habilidades de producción oral y comprensión auditiva. Junto con ello, se pretende contribuir al rendimiento académico de estos grupos de discentes y fortalecer aspectos como la confianza para hablar en público y la autoestima al motivarles a realizar proyectos de diversa índole.

Además de la modalidad preescolar y la modalidad escolar, se facilitan talleres conversacionales en donde la sesión se extiende por una hora más, pues al tomar la modalidad de taller, se han cursado la mayoría de los módulos y una mayor cantidad de horas pretende maximizar la participación conversacional de cada individuo.

A continuación, se presentan las cinco categorías en las que se divide el programa. Estas categorías se realizan por grupos etarios, respetando las distintas etapas de la infancia y las características propias de cada una de dichas etapas.

Tabla 1

Cursos del programa English for Kids

\begin{tabular}{l}
\hline Inglés para preescolar de 3-4 años edad \\
\hline Inglés para preescolar de 5-6 años de edad \\
\hline Inglés para escolar de 7-9 años de edad \\
\hline Inglés para escolar de 10-12 años de edad \\
\hline Taller de Inglés Conversacional para preadolescentes \\
\hline
\end{tabular}

La tabla 1 muestra los diferentes cursos en los que está dividido el programa de inglés para la población infantil. La modalidad de taller trata de abarcar el rango de edad de los 11 a los 13 años. Como ya se mencionó, se encuentra dirigida a estudiantes que han finalizado el programa de año y medio, y desean seguir practicando el idioma para reforzar sus habilidades conversacionales. Una vez finalizado el bloque de talleres, se pasa al programa de adolescentes que está constituido por cuatro cursos enfocados en destrezas lingüísticas específicas.

Los cursos de nivel preescolar pretenden estimular el desarrollo de las habilidades de producción oral y comprensión auditiva mediante técnicas dinámicas que permitan un aprendizaje natural del idioma con ayuda de actividades propias para la edad del grupo discente, lo cual le confiere un papel más activo durante la clase. En el caso de los grupos compuestos por estudiantes de tres y cuatro años de edad, esta suele ser su primera experiencia con el idioma inglés, por lo que la idea de las actividades interactivas que involucren a todo el grupo es que promuevan un ambiente ameno para que el estudiantado coseche experiencias 
favorables con respecto al aprendizaje del idioma. Esto, propiciará el desarrollo de actitudes positivas hacia el idioma inglés en el futuro fomentando bases para un mayor aprendizaje, lo cual tornará más fácil la adquisición del idioma en la educación formal pues "if a person has a positive attitude towards English or the culture in which it is spoken, this person might be motivated to study it [si una persona tiene una actitud positiva hacia el inglés o a la cultura en la que este se habla, es muy probable que se encuentre motivada a estudiarlo]" (LópezMontero, Quesada-Chaves y Salas-Alvarado, 2014, p. 442).

En el caso de los cursos de nivel escolar, se busca reforzar los contenidos vistos en las clases de educación formal, pero con un enfoque comunicativo, en vista de que la mayor parte de la población de estos cursos asiste a centros de educación primaria pública, en los cuales, en ocasiones, por situaciones como grupos sobrecargados de estudiantes, el tiempo efectivo de práctica oral por estudiante puede no ser muy elevado. Entonces, se propone poner énfasis en que cada discente escuche y hable inglés la mayoría del tiempo; y no que lo lea y lo escriba. No obstante, por tratarse de una población con las habilidades de lectoescritura desarrolladas, se aprovechará esta condición para reforzar los conceptos escuchados mediante actividades orales. Este reforzamiento adicional, les será útil para el mejoramiento en la materia en la escuela.

Antes de la presencia de este programa en la región, la oferta de cursos en inglés para niños y niñas era escasa, especialmente a partir de una edad tan corta como los tres años. Si un padre o una madre de familia tenía interés en idiomas para sus hijos e hijas menores de doce años, era casi de carácter obligatorio satisfacerlo fuera de la provincia o, bien, acudir a programas privados de precios altos para la mayor parte de la población. Esto aumentaba los gastos, las limitaciones y privaba a la niñez puntarenense de un avance significativo en su formación bilingüe y en su vida diaria, puesto que el aprendizaje de una nueva lengua es positivo desde muchos puntos de vista. English for Kids ha proporcionado una opción para la familia puntarenense comprometida con el crecimiento cognoscitivo de sus integrantes más jóvenes.

Este programa impulsa el aprendizaje de un idioma desde una edad temprana ya que es una etapa óptima para el aprendizaje de otras lenguas. Sobre esta línea, Quesada-Pacheco (2011) apunta que el mejor momento para enseñar a un niño o niña un segundo idioma es mientras se encuentra aprendiendo la lengua materna y cuanto más pronto los padres y madres de familia expongan a sus hijos e hijas a un segundo idioma, será más fácil para estos y estas reproducir los sonidos propios de esta lengua.

Aunado a esto, los tres años es una edad apropiada para el inicio de la enseñanza de un idioma en este tipo de programas, ya que según Bergeron (2000), es a los tres años en que las personas menores inician una transformación en distintos aspectos, se adquiere un carácter social y se desarrolla el lenguaje: "movimiento, tonos, reflejos, se aproximan poco a poco a sus homólogos en el adulto" (p. 24).

Con el paso del tiempo, la cantidad de cursos impartidos a esta población ha ido en aumento, lo cual ha generado un compromiso cada vez mayor en el seno del programa para mejorar la enseñanza del inglés como lengua extranjera en la población que representa el futuro de la región, es decir, la población infantil.

Este crecimiento puede visualizarse en la tabla 2, que representa la cantidad de cursos impartidos en el primer período de cada año, que es cuando se propone una nueva oferta de cursos. 
Tabla 2

Cantidad de cursos impartidos en I período de cada año.

Tomado de los registros del proyecto

\begin{tabular}{cc}
\hline Año & Cantidad de grupos \\
\hline 2013 & 4 \\
\hline 2014 & 7 \\
\hline 2015 & 9 \\
\hline 2016 & 10 \\
\hline
\end{tabular}

Como se aprecia en la tabla 2, al pasar de los años, la cantidad de cursos impartidos ha aumentado. Se enfatiza en el primer período, pues estos cursos inician únicamente una vez al año; los cursos impartidos el resto del año son la continuidad del programa, por lo que mantiene el mismo estudiantado, a excepción de aquellas personas que ingresen por medio de una prueba de nivelación. Esto demuestra el grado de consciencia que existe en la región sobre la importancia de aprender un nuevo idioma en la etapa de la niñez. Se detallará más sobre estos beneficios, en el siguiente apartado.

\section{Bilingüismo desde la niñez}

Alarcón-Neve (2002) manifiesta que desde la perspectiva psicolingüística "el bilingüismo individual se ha considerado como la posibilidad de un individuo de desenvolverse en dos lenguas" (p. 126). La importancia de comunicarse en la comunidad internacional en más de una lengua es evidente en esta sociedad globalizada y es parte de los retos exigidos a las nuevas generaciones.

La infancia es una etapa idónea para iniciar esta formación lingüística, al respecto, McClandess y Trotter (1984) dicen que: "los niños no se empeñan en aprender un idioma, simplemente lo aprenden" (p. 312). Por otra parte, Brown (2007) menciona la hipótesis del período crítico del lenguaje, la cual establece que existe un período biológicamente determinado en la vida del ser humano en el que los idiomas son adquiridos más fácilmente, después del cual este proceso es más difícil. Esto reforzaría la importancia de aprovechar este momento en la vida de los niños y las niñas.

En este programa, el concepto de bilingüismo se maneja desde lo expuesto por AlarcónNeve (2002). Esta autora explica el concepto de adquisición consecutiva de una lengua, también denominada bilingüalidad consecutiva:

Se da cuando el niño adquiere su segunda lengua en los primeros años de su infancia pero después de que ha adquirido las bases lingüísticas de su lengua materna. Puede ser el resultado de un programa de educación bilingüe porque de manera formal y oficial existe el interés de adquirir una segunda lengua (p. 127) 
Esto es lo que, según Alarcón-Neve (2002), es denominado por Snow en el año 1999, como inmersión lingüística.

Ahora bien, Brown (2007) comenta que los niños y niñas que aprenden dos lenguas simultáneamente las adquieren a través de estrategias similares, pues se encuentran, en esencia, aprendiendo dos lenguas maternas y la llave para el éxito en este proceso está en distinguir contextos separados para ambas lenguas. Según él, aquellas personas que aprenden un segundo idioma en contextos separados son descritas como bilingües coordinadas, pues manejan dos sistemas de significado; por el contrario, las personas que son bilingües compuestos poseen un único sistema de significado desde el cual ambas lenguas operan. Desde su postura, la población infantil, por lo general, no tiene problemas mezclando las lenguas a pesar del grado de separación de contexto para el uso de esas lenguas.

Igualmente, existen una serie de beneficios obtenidos por medio de la instrucción de una nueva lengua. Retomamos las palabras de Vásquez-Carranza (2009) al manifestar que quienes dominan al menos dos idiomas experimentarán un impacto significativo en su desarrollo cognitivo, por lo que aquellas personas con acceso a programas bilingües contarán con mayores posibilidades de sobresalir en el ámbito académico. Esta idea es parte de la visión del programa English for Kids: proporcionar espacios para tales efectos.

Además, Alarcón-Neve (2002) retoma a McLaughlin (1984) al comentar que existe una tendencia a tomar la edad de los tres años "como criterio para separar un bilingüismo temprano de uno tardío" (p.127). En concordancia con esta idea, el programa de cursos libres de inglés de la Sede del Pacífico incentiva una formación bilingüe temprana, para palpar diversos beneficios.

Aunado a lo anterior, el bilingüismo desde una edad precoz conlleva una serie de ventajas para la población infantil que va desde el fortalecimiento de la autoestima hasta aspectos cognoscitivos. En este documento, entiéndase autoestima como "el juicio que efectúan los estudiantes de sí mismos" (Montes de Oca-Rodríguez, 2005, p. 62). En esta línea, Brown (2007) apunta que ninguna actividad cognitiva puede llevarse a cabo sin algún grado de autoestima. De ahí, que sea importante fortalecerla con una práctica docente innovadora y atractiva para las personas discentes. Se detallará más sobre estos beneficios en la población en cuestión más adelante en este ensayo.

\section{Giras académicas como estrategia para fortalecer programas bilingües}

Conscientes de la importancia de la enseñanza de otros idiomas desde edades tempranas y al ser este un programa de cursos libres, desde su seno se realizan diversos esfuerzos por ejecutar una enseñanza innovadora y, por lo tanto, atractiva para las jóvenes personas aprendices. De este modo, se evita la deserción y se alcanza un mayor índice de bilingüismo en la población infantil puntarenense.

Una actividad que ha resultado ser atractiva para el estudiantado y efectiva para la práctica de la lengua meta fuera del aula es la gira educativa. Se realizan giras educativas como estrategia didáctica, tanto a lugares de la provincia como fuera de ella. Su objetivo principal es motivar al estudiantado a desenvolverse en un contexto desconocido aplicando el conocimiento adquirido en clases para maximizar el aprendizaje. 
Tal y como Brown (2007) destaca, hay aprendices visuales que tienden a preferir la información gráfica, aprendices auditivos que prefieren ejercicios de escucha, y aprendices kinestésicos quienes demuestran una preferencia por demostraciones y actividades físicas que involucren movimientos corporales. Por lo tanto, las giras académicas propuestas desde el cuerpo docente procuran abarcar esta gama de habilidades e intereses, para motivar al estudiantado a aprender la lengua inglesa. Esto es de particular importancia en un aula de niños y niñas, indistintamente de la edad en la que se encuentren, ya que tal como afirma Ur (2000), se puede incrementar la motivación y el entusiasmo del estudiantado infantil más fácilmente que la del estudiantado adulto, pero también se puede perder aún más fácil con actividades monótonas sin un fin visible.

Por lo general, la población infantil aprende bien cuando está activa y dicha acción se canaliza en actividades lúdicas. En estas, se encuentran en disposición de invertir su tiempo y esfuerzo, por lo que si se diseñan juegos de forma que esta población produzca lingüísticamente, se convertirán en actividades esenciales en un programa de aprendizaje infantil (Ur, 2000). En el caso de las giras de campo, los grupos estudiantiles tiene la posibilidad de participar en actividades lúdicas fuera del aula, de una manera más libre, a la vez que comparten con compañeros y compañeras de otros cursos y otras edades.

Asimismo, en los niños y niñas con formación bilingüe, cada lengua se desarrolla respondiendo a las condiciones sociales e interactivas del ambiente. Por esta razón, English for Kids visualiza las giras educativas como una oportunidad para que la población participante entre en contacto con el medio que la rodea. La ejecución de dichas giras ha sido de particular importancia dentro del programa, ya que ha demostrado un aumento en la motivación del alumnado para querer participar más activamente con los conocimientos que ha adquirido y las habilidades que ha desarrollado, durante la realización de estas giras didácticas. Con respecto a este tipo de estrategia, Córdoba-Berroa (2008) indica que:

Una excursión o gira educativa pertenece al grupo de actividades y recursos preferentemente de observación, y como todo elemento didáctico, debe llevar una sistematización en su preparación, es decir, plantear objetivos, los contenidos a relacionar con nuestro programa educativo y las actividades previas, durante y posteriores a la visita. (p. 3676)

A lo largo de este programa, se han realizado cuatro giras fuera de las aulas: una en la provincia donde viven los estudiantes y las estudiantes, Puntarenas; y tres más, fuera de ella. Las giras fuera de Puntarenas procuran realizarse una vez al año, como una manera de motivar al estudiantado. Estas son importantes por reafirmar el vocabulario repasado en clase en un ambiente más real y, como ya se ha mencionado, incrementan la motivación de quienes participan, ya que "las giras educativas como estrategia metodológica permiten [sic] a estudiantes y docentes trascender de las aulas" (Gutiérrez-Gutiérrez y Rojas-Núñez, 2014, p. 106). Al tratarse de cursos de inglés, un factor fundamental para el desarrollo de las giras es que los recorridos sean totalmente en la lengua inglesa. De esto se encarga el cuerpo docente, quien mediante su iniciativa propone posibles visitas a lugares dependiendo de los contenidos practicados en el aula.

No obstante, previo a cada gira, el estudiantado ha debido haber participado en por lo menos tres cursos completos del programa; por esta razón, las giras se realizan durante la 
segunda mitad del año, para lograr que el estudiantado forme bases lingüísticas suficientes para desempeñarse en las actividades a realizar durante la experiencia.

A continuación, se explicarán brevemente cada una de las giras realizadas para visualizar el tipo de trabajo que se ha llevado a cabo en ellas y cómo han procurado el alcance de los objetivos del programa.

Primero, con el fin de explotar los recursos que Puntarenas brinda y que en tantas ocasiones son desconocidos por sus mismos habitantes, se visitó el Parque Marino del Pacífico con el objetivo de repasar el vocabulario relacionado a animales y, a la vez, concientizar a los niños y niñas de la importancia de cuidar las especies marinas, tan propias de su contexto inmediato. Posteriormente, se realizó una excursión al Museo de los Niños en la ciudad capital, San José, para profundizar en el estudio de las ocupaciones y profesiones. En esta ocasión, el estudiantado tuvo la oportunidad de desempeñarse como profesional de la medicina, de la mecánica, de la música y otros, a la vez que realizaban actividades en inglés. Una nueva gira didáctica se realizó al Instituto de Biodiversidad, para desarrollar más detalladamente el contenido referente a la flora y fauna, visto en clase. Funcionarios y funcionarias de la institución realizaron talleres en inglés sobre el medio ambiente para los pequeños y las pequeñas desde los tres años hasta los doce años de edad, se dividieron en grupos según su rango etario y se les permitió entrar en contacto con los animales. Otra de las giras académicas se realizó en una lechería, en las faldas del Volcán Poás, en la provincia de Alajuela, para explicar los procesos de producción de la leche y sus derivados, es decir, el enfoque fueron los alimentos.

La escogencia del lugar para cada gira tiene que ver primeramente con la disponibilidad de recorridos o charlas en el idioma inglés y que estos sean relacionados a vocabulario que hayan visto en clase, como los animales, las ocupaciones, el medio ambiente, los alimentos; para que sean identificados por el alumnado. En este caso se pone a prueba su comprensión, ya que escucharán todas las explicaciones principales de parte de un grupo de personas externas a los cursos, y no de quien se encuentran acostumbrados y acostumbradas a escuchar en el aula, la docente.

En cuanto a la producción lingüística en estas giras de parte de cada estudiante, una de las actividades es que las preguntas y comentarios que se generen durante los recorridos se realicen en inglés. Además, las guías de cada lugar a visitar emiten instrucciones en el idioma anglosajón y realizan pequeños juegos interactivos para que el estudiantado conteste en inglés, propiciando un espacio para la comunicación. Las visitas duran de dos a tres horas, dependiendo de cada lugar.

Como actividad posterior a cada gira, deben presentar en la próxima clase, una reacción de lo vivido en la experiencia académica, una pequeña presentación con fotografías del lugar, con los animales que encontraron, con su parte favorita. El desarrollo específico de cada actividad de seguimiento, será según el criterio de cada docente y estará de acuerdo con el nivel del grupo.

Este tipo de actividades tiene gran trascendencia en la vida de los menores y las menores, ya que lo recuerdan como una de sus actividades favoritas aun cuando ya han concluido su programa de seis módulos. Así se ha desprendido del instrumento de evaluación para valorar el desarrollo de los cursos. Gutiérrez-Gutiérrez y Rojas-Núñez (2014) manifiestan con respecto a estas actividades: 
Es así, como convergen los conocimientos construidos en el ambiente áulico con los que construyen al enfrentarse a las diversas situaciones del contexto, es en esta dicotomía, que la estrategia metodológica de las giras, se convierte en una herramienta que reta tanto al profesorado como a los estudiantes. (p. 106)

Además de estas giras, se realizan otras actividades fuera del aula, pero dentro de la universidad, que también han evidenciado un efecto positivo en la población estudiantil, tales como: mini chef por un día, villancicos en inglés, campamentos de inglés, este último tanto para estudiantes activos y activas como para quienes ya hayan finalizado todos los módulos del programa. Una que genera gran expectativa tanto en el cuerpo docente como en el estudiantil, sin duda, la constituye la gira educativa.

\section{Aportes del programa English for Kids para la comunidad estudiantil}

Para determinar los aportes de este programa para sus estudiantes, se toman en cuentan los resultados correspondientes a la evaluación de cursos, realizada entre la sétima y octava semana de clases.

Para dicha evaluación se usan dos instrumentos modificados del instrumento de evaluación de la Vicerrectoría de Acción Social de la Universidad de Costa Rica para actividades de extension docente: uno para las personas encargadas de los niños y las niñas y un instrumento más simplificado para participantes en edad escolar, quienes ya se encuentran en capacidad de proporcionar su propia opinión.

La versión para madres y padres de familia pretende descubrir las razones por las cuales decidieron inscribir a sus menores en los cursos, y si piensan que estas razones se están cumpliendo, si consideran que los objetivos del curso se están alcanzando y si notan mejoría a partir de la participación de sus hijos e hijas en estos cursos en aspectos como la autoestima, motivación, seguridad, niveles de socialización, rendimiento académico y gusto por el idioma inglés y otras lenguas extranjeras.

En el caso del estudiantado, consideramos pertinente conocer el punto de vista de quienes reciben los cursos, ya que se trata de la fuente primaria, por lo que se modificó el instrumento de evaluación original a un lenguaje sencillo que los niños y niñas puedan comprender fácilmente y que proporcione la información suficiente para conocer cómo se estan desarrollando las lecciones. Los aspectos que busca indagar esta versión del instrumento son: el grado de empatía del alumnado hacia su docente, si le gusta o no el idioma y por qué, lo que más le gusta de las clases de inglés, así como lo que menos le gusta.

Entonces, se procede a reseñar brevemente los resultados más relevantes obtenidos de estos instrumentos para visualizar así los aportes puntuales del programa desde dos perspectivas: la de los padres y madres de familia y la del estudiantado.

En primera instancia, a lo largo del tiempo, las personas encargadas de estos grupos de menores han coincidido en la razón por la que deciden matricular los cursos: la importancia de aprender otro idioma, en el caso específico de aprender el idioma inglés, comentan que se trata de una lengua requerida en la vida académica y profesional del alumnado, algo que 
consideran, en la totalidad de quienes han llenado el instrumento, se está logrando; es decir, perciben un aprendizaje de la lengua en estos grupos.

En segundo lugar, de los aspectos en los que manifiestan notar una mejoría debido a los cursos, los siguientes son los elegidos más frecuentemente por la mayoría: primeramente, notan una mayor anuencia o gusto por el idioma inglés y una mayor seguridad en sus hijos e hijas, un mayor grado de socialización y de motivación. Entre los comentarios adicionales en este apartado, una de las personas participantes destaca que su hija "le ha perdido miedo a los profes", "es más sociable y participativa" y que las clases "le alientan a ser más segura".

Por otra parte, el instrumento le pregunta al estudiantado si le gusta el inglés, a lo que la amplia mayoría responde que sí. Ha habido dos casos en los que estudiantes anotan que no les gusta, dando como razón que "les cuesta". También, se indaga sobre las razones por las que les gusta la lengua, esto se realiza mediante una pregunta abierta, con el fin de conocer su percepción sobre el idioma. Las razones mencionadas son las siguientes:

- Gusto por el idioma

- Porque es bonito

- Para viajar y hablar con extranjeros

- Porque sirve para el colegio

- Es divertido

- Para una carrera

- Porque abre puertas

- Porque es el segundo idioma en Costa Rica

- Para jugar videojuegos

- Sirve para siempre, para un futuro

Algo que vale la pena rescatar respecto de estas respuestas es que las relacionadas a un bienestar futuro, provienen de la población de diez a doce años de edad, estas personas parecen proyectarse más a futuro y parecen tener un mayor grado de consciencia sobre el hecho de que se encuentran forjándose un porvenir y que el idioma extranjero contribuye en ese sentido. Independientemente de la edad, sin embargo, las respuestas evidencian una actitud positiva hacia la lengua meta, lo cual está relacionado con la motivación, según lo expresado por López-Montero et al. (2014) cuando afirman que, si una persona posee actitudes positivas hacia un idioma, esto le motivará a aprender este idioma. Esto, a su vez, coincide con los resultados del instrumento para padres y madres de familia que apuntan al factor de la motivación como uno de los que ha mejorado a partir de estos cursos.

De acuerdo con García-Esquivel (2011), la motivación es "algo que influye en nuestra conducta llevándonos a realizar determinados actos para conseguir un determinado fin" ( $p$. 10). Esto es positivo en tanto que contribuye en el estudiantado a cumplir los objetivos de los cursos y a palpar las razones por las que les gusta el idioma inglés. 
En cuanto lo que más les gusta hacer en clases, se les da una lista con las actividades más comunes a desarrollar en el transcurso de las lecciones, además de un espacio para que agreguen cualquier otra que no esté en la lista. Se destacan las tres que se han escogido un mayor número de veces: escuchar canciones, trabajar en grupo y hacer juegos, lo que brinda un panorama del tipo de dinámicas más motivantes para ellos y ellas. Adicionalmente a estas actividades, otras que disfrutan son: cantar, practicar la fluidez, ver videos, realizar giras, y una de las que más se repitió: compartir con amigos. El último aspecto coincide con el mayor grado de socialización que destacan las personas encargadas como uno de los factores que han mejorado a partir de estos cursos, lo que indica que este tipo de experiencias académicas favorecen aspectos más allá de los lingüísticos, el aspecto social se fortalece de manera similar.

En cuanto a la indagación sobre lo que menos les gusta, la pregunta se realizó de forma abierta, demostrando que lo que resulta menos atrayente es realizar presentaciones al frente y, en menor cantidad, que no se les hable en español. Este último caso puede tornarse algo frustrante cuando no se comprende todo lo que se dice, por lo que se trata de que las explicaciones siempre vengan acompañadas de lenguaje corporal para faciltar la comprensión del estudiantado evitando el uso de la lengua materna.

Estos resultados aportan insumos de gran relevancia para el programa, ya que indican los avances desde los puntos de vista de las personas implicadas: aspectos como la motivación, mejor socialización y mayor seguridad contribuyen a formar seres humanos integrales, además de facilitar las herramientas para aprender un nuevo idioma. También, descubrir que existe un gusto por parte de los niños y niñas hacia esta lengua es gratificante, puesto que es un indicador de que se está caminando hacia los objetivos establecidos para el programa; como ya se ha dicho, el que el estudiantado perciba positivamente la lengua meta facilita el aprendizaje y esto es perceptible al conocer las razones que el mismo cuerpo estudiantil aporta para la adquisición del idioma.

El conocer las actividades más atractivas en el desarrollo de la clase, así como las que atraen menos, permite conocer cuáles deben redireccionarse para trabajar mejor en ellas y cuáles deben reforzarse o incluirse.

Cada niño y niña ha recibido un impacto positivo por la oportunidad de tener acceso a la adquisición de un nuevo idioma, permitiéndoles un mayor crecimiento social, el cual es desarrollado al tener contacto con otras personas en diferente lengua. Por otra parte, el proyecto abre sus puertas a una sociedad que requiere de mayor motivación para triunfar y para acceder a mejores oportunidades.

Aunado a lo anterior, al usar una escala de evaluación menos rígida que la utilizada en la educación formal (son cursos participativos), la población estudiantil no siente la presión de obtener una nota para saber que están aprendiendo, lo que aumenta la motivación y favorece la autoestima, permitiendo un mayor desarrollo de la destreza lingüística y la capacidad verbal. Además de estos aspectos, se encuentra el desarrollo de la creatividad, ya que mediante los cursos es el alumnado quien propone las actividades a realizar en las presentaciones especiales, lo que al mismo tiempo incentiva el desarrollo de las habilidades de resolución de problemas y una mayor independencia y autonomía. 


\section{Conclusiones}

El proyecto de Idiomas, mediante su programa English for Kids, es una herramienta educativa que va dirigida a impulsar a la provincia de Puntarenas en nuevos retos educativos, sociales y culturales. Este programa fue diseñado y formado con el fin de desarrollar en la niñez puntarenense el gusto por aprender un nuevo idioma tomando en cuenta su punto de vista como participantes para determinar lo que más les motiva y facilitar que desarrollen una actitud positiva ante el idioma en el futuro y, por lo tanto, facilite su aprendizaje en la educación formal y en cualquier otra experiencia que tengan con la lengua, fomentando así un mejor rendimiento académico y desarrollando otras áreas de importancia en el desarrollo del ser humano como la socialización y autoestima.

La exposición del alumnado al idioma inglés debe ser la más alta posible al tiempo que aprenden su lengua materna. Mientras el individuo empieza a aprender inglés, como se ha expuesto anteriormente, hay otras capacidades desarrollándose simultáneamente como la destreza lingüística, la capacidad verbal, la creatividad, las habilidades de resolución de problemas, la seguridad en sí mismos y en sí mismas, por lo que el apoyo recibido desde sus hogares para la realización de sus proyectos, el repaso de la materia y la asistencia a clases es vital.

Resulta de suma importancia recalcar que la extensión universitaria no busca la distención de clases; por el contrario, busca la unificación de una provincia para sobresalir y prevalecer a pesar de cualquier dificultad económica, social o moral. El programa se forjó con el interés de ayudar a consolidar una educación enriquecedora, y explotarla al máximo a pesar de las limitaciones del entorno.

La adquisición de una nueva lengua es el viaje a descifrar una cultura diferente y renovada, por lo tanto, cada niño y niña que cuente con el apoyo de sus progenitores aunado a programas de acción social que le permitan un crecimiento sólido podrá encabezar un futuro lleno de habilidades cognoscitivas y oportunidades lingüísticas.

English for kids es y será para el bienestar de la comunidad puntarenense, y está libre de todo carácter discriminatorio y estatus social. Es un programa que se compromete a mantener la constante búsqueda de nuevas estrategias pedagógicas, didácticas, motivacionales y culturales que permitan el crecimiento y buen desarrollo cognitivo del estudiantado, tomando en cuenta su perspectiva.

No se pretende con este proyecto, que el estudiantado sea completamente bilingüe al finalizar cada curso, pues para ello se necesita una mayor exposición fuera del contexto áulico, por lo que se planean actividades como giras didácticas y otros proyectos fuera de clase, pero sí pretende sumarse a los esfuerzos promovidos desde las políticas educativas bilingües del país para que los ciudadanos y las ciudadanas del futuro y de cualquier región de la nación, sean seres humanos con una formación integral, la cual se forja desde el aprendizaje de cualquier materia, en este caso, desde el idioma inglés como lengua extranjera.

En resumen, el avance de la provincia de Puntarenas depende de las personas que la habitan; por tal razón, los programas de mejoramiento que se puedan brindar en dicha comunidad cumplen un papel relevante. Por tanto, el programa English for Kids pretende fomentar el estudio del idioma inglés en la población infantil con el fin de aportar en el mejoramiento a la calidad de vida de las personas puntarenenses a través de la extensión docente. 


\section{Referencias}

Alarcón-Neve, L. (2002). Bilingüismo y adquisición de segundas lenguas: Inmersión, Sumersión y Enseñanza de Lenguas Extranjeras. En Universidad Autónoma de Querétaro. Simposio CONCYTEQ La investigación y el desarrollo tecnológico en Querétaro (pp.124-133). Universidad Autónoma de Querétaro. Recuperado de http:// luisalarcon.weebly.com/uploads/2/7/8/4/2784997/bilingismo y adquisicin de segundas lenguas- inmersin sumersin y enseanza de lenguas extranjeras.pdf

Bergeron, M. (2000). El desarrollo psicológico del niño (4a ed.). Madrid: Morata, S. L.

Brown, D. (2007). Principles of Language Learning. [Principios del aprendizaje de idiomas] $\left(3^{\mathrm{a}}\right.$ ed.). Nueva York: Pearson Education.

Córdoba-Berroa, A. (2008). La importancia de las excursiones educativas como estrategia didáctica en la enseñanza de la historia. Diálogos Revista Electrónica de Historia. (Número especial), 3662-3692. Recuperado de: http://www.historia.fcs.ucr.ac.cr/ articulos/2008/especial2008/articulos/12Teoria/150.pdf

Córdoba, P., Coto R., y Ramírez, M. (2005). La enseñanza del inglés en Costa Rica y la destreza auditiva en el aula desde una perspectiva histórica. Revista Actualidades Investigativas en Educación, 5(2), 1-12. Recuperado de http://revista.inie.ucr.ac.cr/index. php/aie/article/viewFile/71/70

García-Esquivel, R. (2011). Estudio sobre la motivación y los problemas de convivencia escolar. (Tesis de maestría). Facultad de Ciencias de la Educación, Universidad de Almería. Recuperado de http://repositorio.ual.es/jspui/bitstream/10835/1179/1/Garcia Esquivel Raquel.pdf

Gutiérrez-Gutiérrez, M., y Rojas-Núñez, P. (2014). Las giras educativas: Una estrategia metodológica para la articulación teoría y práctica. Revista Inter Sedes, 15 (31), 99-114. Recuperado de http://revistas.ucr.ac.cr/index.php/intersedes/article/view/16016/15417

Harmer, J. (2007). How to teach english. [Cómo enseñar inglés]. Inglaterra: Pearson Education.

Hasbún-Hasbún, L. (2007). Algunas razones por las que nuestro mundo debería ser multilingüe. Revista de Lenguas Modernas, 6, 109-117.

Instituto Nacional de Estadística y Censos. (INEC). (2011). Censo 2011. Recuperado de http:// www.inec.go.cr/Web/Home/GeneradorPagina.aspx 
López-Montero, R. (2014). Puntarenas hacia el camino del multilingüismo: Impacto del Proyecto de Idiomas de la Sede del Pacífico de la Universidad de Costa Rica. Revista Inter Sedes. 15 (30), 132-150. Recuperado de http://revistas.ucr.ac.cr/index.php/intersedes/ article/view/14873/14150

López-Montero, R, Quesada-Chaves, M., y Salas-Alvarado, J. (2014). Social factors involved in second language learning: a case study from the Pacific Campus, Universidad de Costa Rica [Factores sociales en el aprendizaje de un segundo idioma: El caso de la Sede del Pacífico de la Universidad de Costa Rica]. Revista de Lenguas Modernas, 20, 435-451.

Marín-Arroyo, E. (2012). Cronología de la enseñanza del inglés en Costa Rica durante el Siglo XX. Revista Comunicación, 21(1), 4-15. Recuperado de http://revistas.tec.ac.cr/index.php/ comunicacion/article/viewFile/809/723

McCandles, B. y Trotter, R. (1984). Conducta y desarrollo del niño ( $3^{\mathrm{a}}$ ed.). México: Nueva Editorial Interamericana.

Montes de Oca-Rodríguez, R. (2005). Autoestima e idioma inglés: Una primera discusión. Revista Educación, 29 (1), 59-71. doi: http://dx.doi.org/10.15517/revedu.v29i1.2023

Montrul, S. (2013). El bilingüismo en el mundo hispanohablante. Reino Unido: Wiley-Blackwell.

Quesada-Pacheco, A. (2011). Issues on raising a bilingual child in Costa Rica: a myth or a reality? [Estado de la cuestión: Crianza de un niño o una niña bilingüe en Costa Ricauna realidad o un mito] Revista Actualidades Investigativas en Educación, 11(2), 1-21. doi: http://dx.doi.org/10.15517/aie.v11i2.10192

Ur, P. (2000). A Course in language Teaching. Practice and theory. [Un curso en la enseñanza de idiomas. Práctica y teoría], Cambridge University Press.

Vásquez-Carranza, L. (2009). Cognitive advantages of balanced bilingualism [Ventajas cognitivas de un bilingüismo equilibrado]. Revista Pensamiento Actual, 9 (12-13): 69-78. Recuperado de http://revistas.ucr.ac.cr/index.php/pensamiento-actual/article/ view/2837/2759

Universidad de Costa Rica. (s.f.). ¿Qué es acción social? Recuperado de http://accionsocial.ucr. ac.cr/accion-social

Zhizhko, E. A., y Koudrjavtseva-Hentschel, E. (2011). Bilingüismo natural como fenómeno cultural y su impacto en la educación terciaria transfronteriza. Innovación Educativa, 11 (56), 50-56. Recuperado de http://www.redalyc.org/articulo.oa?id=179421438005 